\title{
Biliary Intraepithelial Neoplasia-1
}

National Cancer Institute

\section{Source}

National Cancer Institute. Biliary Intraepithelial Neoplasia-1. NCI Thesaurus. Code C67491.

Biliary intraepithelial neoplasia characterized by the presence of mild epithelial atypia. 